\title{
Broadening the perspective: Epistemic, social, and historical aspects of scientific modelling
}

Kuorikoski, J.

2015

Kuorikoski , J \& Marchionni , C 2015 , ' Broadening the perspective: Epistemic, social, and historical aspects of scientific modelling ', Perspectives on Science, vol. 23 , no. 4 , pp. 381-385. https://doi.org/10.1162/POSC_e_00179

http://hdl.handle.net/10138/234268

https://doi.org/10.1162/POSC_e_00179

publishedVersion

Downloaded from Helda, University of Helsinki institutional repository.

This is an electronic reprint of the original article.

This reprint may differ from the original in pagination and typographic detail.

Please cite the original version. 


\section{PROJECT MUSE'}

Broadening the Perspective: Epistemic, Social, and

Historical Aspects of Scientific Modelling

Jaakko Kuorikoski, Caterina Marchionni

Perspectives on Science, Volume 23, Number 4, Winter 2015, pp. 381-385

(Article)

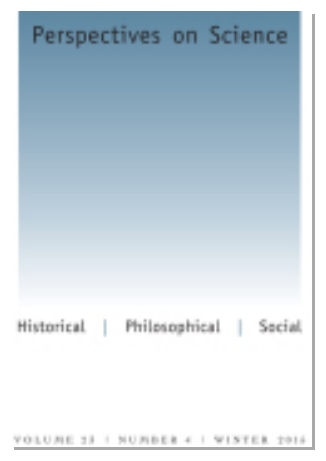

Published by The MIT Press

$\Rightarrow$ For additional information about this article

https://muse.jhu.edu/article/601009 
Broadening the Perspective:

Epistemic, Social, and

Historical Aspects of

Scientific Modelling

\section{Jaakko Kuorikoski}

University of Helsinki

Caterina Marchionni

University of Helsinki

\section{Introduction}

The recognition that models and simulations play a central role in the epistemology of science is about fifteen years old. Although models had long been discussed as possible foundational units in the logical analysis of scientific knowledge, the philosophical study of modelling as a distinct epistemic practice really got going in the wake of the Models as Mediators anthology edited by Margaret Morrison and Mary Morgan (1999). In spite of the broad agreement that in fact much of science is model-based, however, there is still little agreement on pretty much anything else. What are models? Are they representations or fictions, abstract entities or concrete artifacts? Which functions do they play? Can they explain, provide confirmation to hypotheses, or are they mere heuristic devices? If they have independent epistemic power, where does it come from? Moreover, arguments in favor or against alternative positions are often drawn from case studies of particular modelling or simulation practices. Finally, those philosophical accounts that aim at generality often tend to be "deflationary" in spirit since, it is claimed, various kinds of models do not share enough in common to warrant a substantive and yet unified view.

How then is progress in understanding the epistemology and ontology of models to be achieved? Several routes are possible. One route is to devote further philosophical effort into the development of a general account of scientific models, their ontology and epistemology. Another is to examine more, and more diverse, cases of scientific models and aim for better taxonomies and informative generalizations. Yet another is to 
broaden the range of theoretical resources so as to include cognitive, social and historical perspectives on models and modelling. ${ }^{1}$ In this special issue, comprising a selection of papers presented at the conference "Models and Simulations 5" (Helsinki, 2012), all three routes are explored. This introduction is an attempt to highlight the ways in which the various perspectives can work productively together.

\section{The Epistemic Role of Models}

Models are means of surrogate reasoning: we learn about the systems modelled by examining and manipulating an epistemic artifact purposefully constructed to "stand in" for the system. If models can be thought of representing their target, the way in which the model functions as a stand in for its target can hardly be understood independently of its use and purpose. More generally, pragmatic factors are indispensable in defining what the model is a model of as well as its accuracy or success (e.g. Suárez 2004). Recent philosophical interest in modelling was mostly sparked by the realization that models are in many ways autonomous with respect to theory, the unit of knowledge long held to be foundational for science. Models are neither straightforwardly derived from theory nor simply abstracted from observation and experiment.

In their representational capacity models can be thought of as analogous to maps in that they have both a purely iconic or mimetic quality as well as a conventional element. Lehnard (this issue) explores features of computational models brought forth by the map analogy in the case of nanotechnology. First, Lehnard argues that the results of computational modelling do not produce a global representation, but are conceived of as parts of "virtual atlases" made out of local, purpose-dependent maps, which do not fit neatly together. The success of maps does not rest on how accurately they represent their target, but on whether they provide correct predictions (or retrodictions) and suggest effective intervention. Second, maps and computational models are products of negotiation: in the historical past, map making involved negotiation between, say, the cartographer and his/her informants, computational modelling results from a process of negotiation between theory, simulation, and experiment (on the interaction between theory, models and experiments see also Barwich, this issue). The third feature is 'locality': the simulation model is not straightforwardly derived from general laws nor does its success

1. This kind of development possibly reflects a wider trend within the philosophy of science, as witnessed by the increasing attention to social epistemology, integrated history and philosophy of science, and philosophy of science in practice. 
in one situation and under certain conditions imply success in others; in this sense it is local rather than general.

The epistemic justification of different kinds of models need not be the same across the board. Some models are justified in terms of the inferences about a target system they allow a competent user to draw, other models instead are better conceived of as means to "truer theories" (e.g., Wimsatt 2007 , Ch. 6) and do not directly connect to any target system or situation. The former issue has been mainly debated in relation to traditional epistemic notions such as prediction and explanation, but other concepts have also been used to capture the epistemic contribution of models, such as understanding and learning (Grüne-Yanoff 2009). But, are such concepts better understood than explanation or prediction? And how do such "psychological" notions differ from these more traditional epistemic concepts?

Claveau and Vergara (this issue) attempt to operationalize the notions of learning from models by resorting to more traditional epistemological considerations. They propose to understand learning as the process of arriving at new knowledge-intended in the classical sense of justified true beliefs - and come up with a positive verdict: models can indeed serve an evidential role in providing justification for beliefs, a revealing role in suggesting new hypothesis, and a stimulating role in revealing new evidential connections in the body of already accepted beliefs.

The evidential role of theoretical models - whether they provide genuine justification or confirmation over and above already accepted empirical facts - is arguably the major point of contention in the philosophical debate. Instead, the heuristic role of models has been largely taken as unproblematic and has been subject to little philosophical scrutiny. But is it really the case that models are (if nothing else) useful heuristic device, and if so, in virtue of what? Armin Schulz (this issue) proposes to measure heuristic power in terms of new results a model or a simulation generates. On this measure, however, that is not likely to produce genuinely new results because modellers have incentives to employ mostly familiar mathematical structures. Simulations fare slightly better, but their heuristic potential is not as obvious as it might seem, since the incentives are, again, in favour of using already existing simulation platforms. Schulz argues that the preferential use of known structures and platforms means that most modelling results are already foreseen, at least by the modellers themselves.

\section{Modelling As a Social and Historical Activity}

Scientific modelling is part and parcel of an intertwined set of scientific practices, ranging from software development to empirical testing and 
experimentation. Even standard methodologies, such as robustness analysis, appear to essentially require the epistemic community of modellers rather than the individual scientist in order to fulfil their rationale. From this perspective, the social structure of modelling activity is likely to be relevant to the understanding of the epistemic and heuristic performance of models. The social embeddedness of modelling also implies that the history of any particular modelling exercise is ever present in shaping the collective practices of building, using, and evaluating models.

This latter aspect is explored in Barwich's article (this volume). In engaging with the history of olfactory research, she argues that representing with models happens together with methods of experimentation, data collection and analysis within a complete experimental system. This means that there is a certain self-vindicating element in at least those modelling enterprises which are closely tied to experimental practice: a model develops together with the experimental methods used to test and calibrate it in a mutually re-enforcing manner. Hence, rival models cannot be simply compared against data, because a model that is already well established enjoys an advantage in that most of the existing means of data production have been moulded to serve that model. This leads to a certain amount of inertia in model-based research and implies research and implies that the epistemic evaluation of models has to be, at least to some extent, historical.

Modelling requires expertise and the increasing importance of modelling has consequences for the division of scientific labour. Beyond traditional disciplinary boundaries, skills in using specific model templates will likely play an ever-larger role in the way knowledge production is organized (cf. Humphreys 2007). Matt Spencer (this issue) addresses the implications of large-scale computational science to the social organization of cognitive labor. These implications are not only of sociological interest, but have broader social epistemological significance. Spencer broadens the perspective of models as epistemic artifacts by not only considering the material aspects of computer simulation, but by focusing also on the social scaffolding required by these artifacts. For software to function as a versatile and effective platform for computational modelling, to be not just a program but a full programming systems product, it has to be created and maintained by institutionalized social practices of testing, approving, validating, and documenting. Although this increased "bureaucracy" might feel like an unnecessary and even stifling burden for those engaged in computational modelling, it may be necessary to ensure the proper functioning of the epistemic tools used. Combating the brittleness of the code is an epistemically significant task underlying all of computational science and brings with it new demands for the social structures of science. 


\section{Moving Forward}

We believe that broadening the philosophical perspective so as to include its social and historical dimensions is a promising way forward for understanding scientific modelling. ${ }^{2}$ Increasing the breadth of our theoretical resources and closely engaging with relevant empirical research also gives us a better chance of making philosophical reflections relevant to practicing modellers as well as to the end users of model-based knowledge. Purely historical or sociological case studies cannot answer all the epistemological or practical questions concerning the use of model-based knowledge. Arguing for the superiority of one modelling framework over another and assessing the epistemic and practical value of model-based knowledge require justification based on epistemological principles, not only on historical facts or on observations about social processes of model production. Practical relevance cannot be achieved solely with a priori philosophical reflections either. Therefore, a broadly philosophical outlook on the practice of modelling is a good platform for integrating empirical findings from cognitive science, sociology and history of science in such a way as to generate systematic and applicable insights about this central epistemic activity.

\section{References}

Grüne-Yanoff, T. 2009. "Learning from Minimal Economic Models." Erkenntnis 70: 81-99.

Humphreys, P. 2007. Extending Ourselves: Computational Science, Empiricism, and Scientific Method. Oxford: Oxford University Press.

Morrison, M. \& Morgan, M., eds. 1999. Models as Mediators: Perspectives on Natural and Social Science. Cambridge: Cambridge University Press.

Suárez, M. 2004. "An Inferential Conception of Scientific Representation." Philosophy of Science 71: 767-779.

Wimsatt, W. C. 2007. Re-engineering Philosophy for Limited Beings: Piecewise Approximations to Reality. Cambridge, MA: Harvard University Press.

2. A more substantial engagement with cognitive science would also undoubtedly enrich our understanding of model-based reasoning. This is true especially if we take learning more broadly, over and above justification, to be a philosophically relevant aspect of modelling. 